\title{
Effects of different Diaphragm Breathing Methods on the Diaphragm Thickening Ratio and Pulmonary Function in Young Adults
}

\author{
Tae-Won Ha, PT • Myung-Mo Lee, PT, $\mathrm{PhD}^{1 \dagger}$ \\ Department of Physical Therapy, Graduate School of Health and Medicine, Daejeon University \\ ${ }^{1}$ Department of Physical Therapy, Daejeon University
}

Received: October 12, 2018 / Revised: October 16, 2018 / Accepted: October 16, 2018

(c) 2019 J Korean Soc Phys Med

\section{| Abstract |}

PURPOSE: This study examined the effective impact of self and resistive and ultrasound-biofeedback diaphragm breathing on the pulmonary function and diaphragm thickening ratio of young adults.

METHODS: Thirty normal adults were assigned randomly to three experimental groups (self- diaphragm breathing $(\mathrm{n}=9)$, resistive-diaphragm breathing $(\mathrm{n}=11)$, ultrasoundbiofeedback diaphragm breathing $(\mathrm{n}=10))$. Each group participated for 15 minutes for times with a two minute rest between two sets. The subjects were assessed using the preand post- diaphragm thickening ratio and the pulmonary function (forced vital capacity, forced expiratory volume at one second, maximal voluntary ventilation, and respiratory rate) on the thirty subjects. A paired t-test was to determine the difference between before and after the experiment in each group of diaphragm breathing before and after the exercises.

†Corresponding Author : Myung-Mo Lee mmlee@dju.kr, https://orcid.org/0000-0002-2192-1701

This is an Open Access article distributed under the terms of the Creative Commons Attribution Non-Commercial License (http://creativecommons.org/licenses/by-nc/3.0) which permits unrestricted non-commercial use, distribution, and reproduction in any medium, provided the original work is properly cited.
One-way ANOVA was used to determine the differences between the groups.

RESULTS: The forced vital capacity and maximal voluntary ventilation measurements revealed a significant difference in the resistive-diaphragm breathing group than the other two groups. On the other hand, there was no significant difference between the self-diaphragm breathing and ultrasound-biofeedback breathing groups.

CONCLUSION: The resistive-diaphragm breathing group showed greater improvement in the pulmonary function than the other two groups. Therefore, resistivediaphragm breathing will improve the pulmonary function on normal young adults.

Key Words: Breathing exercise, Pulmonary function, Diaphragm breathing

\section{Introduction}

Respiration is performed by neurological control and the action of the respiratory muscles that takes in oxygen and releases carbon dioxide[1]. For normal breathing, the action of the respiratory muscles should be spontaneous or involuntary, depending on breathing and exhaling[2].

The diaphragm plays a major role in inspiration and 
an external intercostal muscle that is related to the expansion of the heart and regular breathing. The serratus posterior, intercostal muscles, and trapezius, levator scapulae, which spread the spine, are the accessory muscles associated breathing. In addition, the pectoralis major, pectoralis minor, and sternocleidomastoid are involved in deep inspiration[3].

After inspiration, expiration occurs passively due to the difference in pressure between the lung and external pressure. In the accessory expiration muscle, there are the transverse abdominis, external $\cdot$ internal oblique abdominis, internal intercostalis, and rectus abdominis. These muscles work with effort or deep expiration[4].

Degraded breathing muscles can lead to a weakened cardiopulmonary capacity and a lack of oxygen. This results in a decrease in cardiopulmonary volume due to a reduction of the oxygen metabolism, which reduces the elasticity of the chest, leading to a diminished pulmonary function and respiratory control[5]. Air stacking exercises can improve the lung capacity and exercise tolerance significantly in elderly adults[6]. In addition, the ability to remove cough or phlegm is reduced, which can accumulate secretions in the airway, leading to a range of respiratory diseases, such as pneumonia[1].

Various breathing methods used to increase diaphragm breathing involve improving the strength and endurance of the respiratory muscles[7]. Self-diaphragm breathing training, also called diaphragm breathing training, is the main method of breathing, where the upper and lower movements are caused by a contraction of the diaphragm. As a person breathes, the diaphragm contracts down and becomes flat, causing the abdomen to swell. During exhalation, the curtain and abdomen return to their original states[8]. This breathing method is used to exchange effective oxygen for carbon dioxide, and it helps stabilize the body and improve the pulmonary function. In addition, it is a breathing method that can be applied easily in everyday life because it has no side effects and is easy to learn and train[9].

Resistive diaphragm breathing training is simply a method of intervention to increase the inspiratory capacity, which is based on the principle of overloading and applies a load to the supporting muscles of the diaphragm and inspiratory respiratory muscles[10]. Resistive diaphragm breathing training is used widely as an intervention for strength and endurance because a weakening of the inspiratory muscle interferes with the motor performance and breathing skills[11].

Feedback breathing training is a training method that self-modifies and repeats the breathing method based on information provided in real time during a specific task. This is an effective method of breathing training for the elderly or patients with respiratory diseases who cannot use the respiratory muscles correctly because it can induce effective and normal movement of the respiratory muscles by continuously stimulating their ability to control breathing exercises[12].

Kwon et al.,[13] reported a decrease in the thickness of the abdominal oblique muscle and an increase in the thickness of the transverse abdominal muscle through an abdominal hollowing method with ultrasonic image feedback. Nam[14] reported that diaphragm exercises using real-time ultrasound feedback exercises produced significant improvements in disposal for young women with limited chest mobility.

Despite the clinical importance of diaphragm breathing, there are difficulties in learning about unfamiliar breathing methods when applying breathing exercises to the elderly or patients after respiratory diseases. Most of all, it is difficult to recognize the precise contraction of the diaphragm and simple and repetitive respiratory patterns can result in the incorrect use of respiratory muscles. This study examined the changes in the pulmonary capacity and the rate of contraction of the diaphragm by dividing diaphragm breathing training into three groups: selfdiaphragm breathing groups, resistive diaphragm breathing 


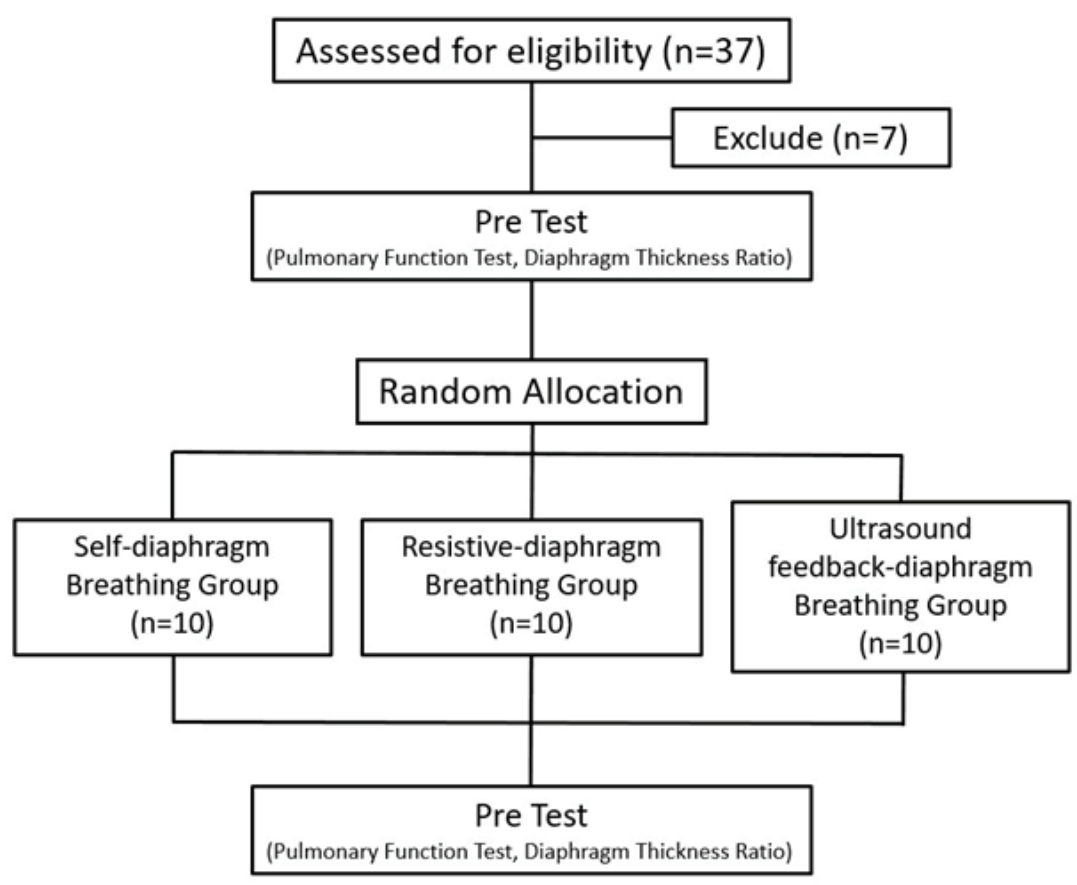

Fig. 1. Flow chart of this study

groups, and real-time ultrasound feedback diaphragm breathing groups. In this study, it was hypothesized that there will be more significant improvements in the pulmonary function and contraction ratio of the diaphragm in a way that the breathing training method provides feedback on the use of the respiratory muscles.

\section{Method}

\section{Participants}

A total of 37 healthy adults were recruited at D University in D city. The selection criteria were those without neurological or musculoskeletal disorders, those without respiratory or circulatory system disorders, those without congenital thorax deformation or ribs, and those who did not have regular surgical diseases. Prior to the experiment, all participants were asked to explain the purpose and procedure of the study. All participants signed an informed consent form for voluntary study participation.
This study was a three-group pretest-posttest design to determine the change in pulmonary function and rate of diaphragm contraction according to the diaphragm breathing method. Of the 37 subjects initially selected, seven did not meet the selection criteria, giving a final total of 30 subjects. The selected subjects were pre-assessed followed by a random assignment program to either the self-diaphragm breathing group (SDBG, $n=10$ ), resistivediaphragm breathing group (RDBG, $\mathrm{n}=10)$, or real-time ultrasound feedback diaphragm breathing group (UFDBG, $\mathrm{n}=10)$ (Fig. 1).

The SDBG conducted training by placing one hand on the chest and the other on the abdomen, feeling the swelling of the stomach and the movement of the chest. The RDBG provided resistance to breathing by placing a $3-5 \mathrm{~kg}$ sandbag on the abdomen in the supine position. Similar to the SDBG, the RDBG applied breathing exercises to recognize that the abdomen would elevate and depress without chest movement in response to the resistance of the weight of 


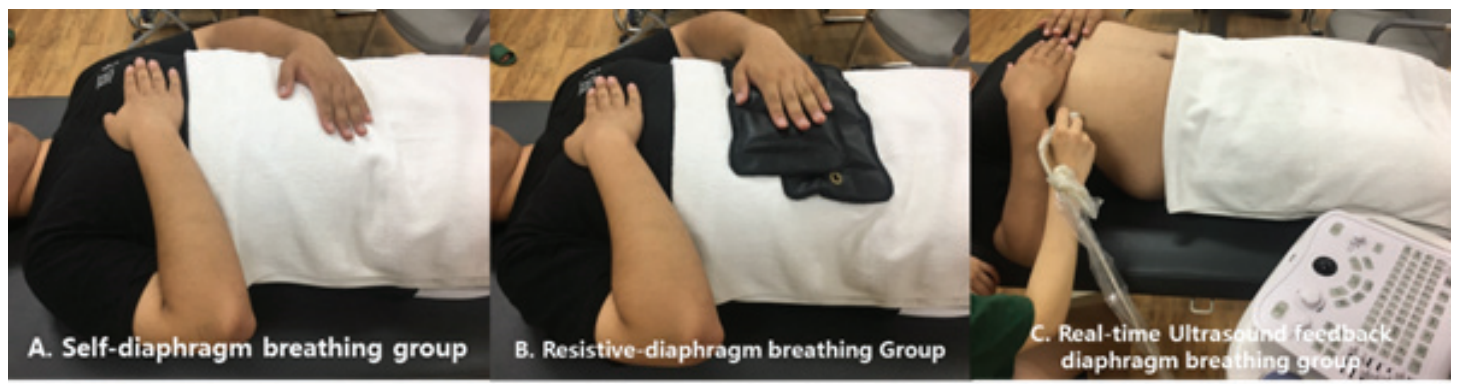

Fig. 2. Three groups according to diaphragm breathing method

the sandbags during inspiration and expiration. The sandbag weight was applied differently for each subject and at levels 11 to 13 of 'Rating of Perceived Exercise (RPE)'. The UFDBG applied breathing exercises while in the supine position, visually identifying live motion images of the diaphragm provided by the tester using a real-time ultrasound imaging device. The breathing exercise for each group were conducted in two sets for 15 minutes with a five-minute break between the sets. A sufficient 10 minute break was allowed after completing the breathing exercises. All diaphragm breathing exercises were conducted under the guidance and supervision of one examiner (Fig. 2).

\section{Measurement and Procedure}

\section{1) Pulmonary function test}

A spirometer (SPIROMETER HI-101, Chest M.I., Inc., Japan) was used to test the pulmonary function. The subjects leaned against the wall with their nose closed, put a mouthpiece in their mouth, and performed a lung function test as directed. The FVC (Forced vital capacity), FEV1 (forced expiratory volume in 1second), MVV (maximal voluntary ventilation), and RR (respiratory rate) were measured and recorded during the pulmonary function test. Prior to the measurement, the tester was fully trained through a description and demonstration of the testing method. The highest value was recorded after three measurements.

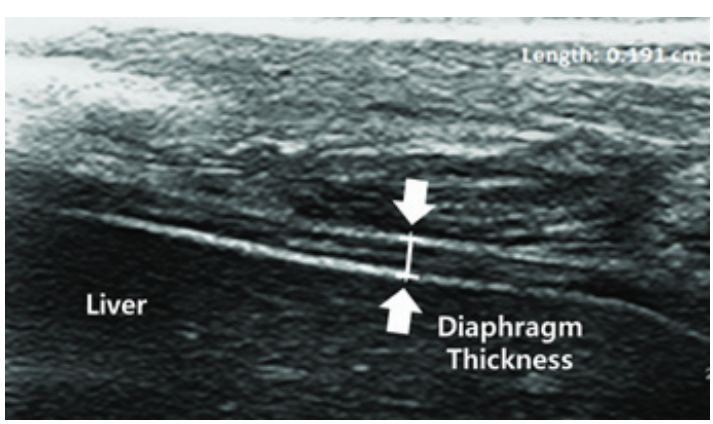

Fig. 3. Diaphragm on Real-time ultrasound imaging

\section{2) Diaphragm thickness}

A real-time ultrasound imaging device (Digital Ultrasound Diagnostic Imaging System DP-6600, Mindray, Indonesia) was used to measure the diaphragm thickness and rate of change according to the diaphragm breathing training method. In the sitting position, a pen was placed on the chest wall between 8 and 9 ribs on the right side of the target. The probe was then placed vertical in contact with the marked area with the patient in the upright position to confirm the contraction in the plane image. The thickness of the diaphragm is the distance of two parallel lines that appear white in the middle of the breast film and in the middle of the diaphragm, causing maximum breathing of the subject, which was measured three times and averaged [15](Fig. 3).

To measure the diaphragm thickness during diaphragm breathing, the diaphragm thickness was measured at the time of maximum inhalation and maximum expiration with 
the nose closed by hand. The standardized contraction of the barrier used by Sarwal et al.[15] was applied.

$$
\mathrm{TR}=\frac{\begin{array}{l}
\text { Tinp.con }(\text { thicknessaed }- \text { inspiration })- \\
\text { Texp.con }(\text { thicknessated }- \text { expiration })
\end{array}}{\text { Texp.con }(\text { thicknessated }- \text { expiration })}
$$

\section{3) Statistical analysis}

The data were analyzed using the SPSS ver. 20.0 program (SPSS Inc., Chicago, II, USA). The homogeneity test of the general characteristics of the subjects is expressed as the mean and standard deviation after applying a paired-t-test, and a normal distribution was obtained through a Shapiro-Wilk normality test. Comparisons between pre-post intervention groups were made using a paired t-test. One-way repeated analysis of variance (ANOVA) was used to compare the pre- and postinterventions. A Bonferroni correction was used as a post-test. To verify the statistically significant differences, the significance level a was set to 0.05 .

\section{Result}

Finally, 10 subjects in each group performed the post-assessment. Table 1 lists the general characteristics of the subjects; there was no difference in the general characteristics between the groups.

Table 2 lists the results of the pulmonary function test among the groups according to the breathing training method, and the pre-measurement values were all homogenized among the groups. As a result of the before-after comparison within the group, the SDBG showed no significant changes in any of the pulmonary function tests. The RDBG and showed significant statistically significant improvements in the FVC and MVV.

A comparison of the differences in pulmonary functions among the groups revealed significant differences in the FVC and MVV. The RDBG showed significant improvements in the FVC and MVV compared to the SDBG $(p<.05)$.

The thickness and retraction rate of the air intake and the pre- and post-group comparisons were similar in the three groups, and there was no significant difference in the amount of variation among the groups (Table 3).

\section{Discussion}

This study compared the changes in pulmonary function and the rate of diaphragm contraction according to the diaphragm breathing training method applied to normal adults. As a result, the RDBG and UFDBG showed immediate improvements in the FVC and MVV. Resistivediaphragm breathing training in significant improvements in the FVC and MVV compared to self-imposed breathing exercises.

Self-diaphragm breathing exercises have been proposed

Table 1. Characteristics of the subject

\begin{tabular}{ccccc}
\hline & SDBG $\left(\mathrm{n}_{1}=10\right)$ & PDBG $\left(\mathrm{n}_{2}=10\right)$ & UFDBG $\left(\mathrm{n}_{3}=10\right)$ & $\chi^{2} / \mathrm{p}$ \\
\hline Age (years) & $23.003 \pm 2.454^{\mathrm{a}}$ & $21.334 \pm 1.069$ & $22.003 \pm 2.002$ & .145 \\
Height $(\mathrm{cm})$ & $163.778 \pm 5.541$ & $162.665 \pm 5.540$ & $167.503 \pm 5.891$ & .114 \\
Weight $(\mathrm{kg})$ & $61.059 \pm 11.821$ & $53.752 \pm 5.427$ & $59.200 \pm 7.284$ & .122 \\
Sex (Male/Female) & $(3 / 7)$ & $(1 / 9)$ & $(3 / 7)$ & .475 \\
\hline
\end{tabular}

${ }^{\mathrm{a}}$ Mean \pm standard deviation

SDBG : Self-diaphragm breathing group, PDBG : Passive-diaphragm breathing group,

UFDBG : Real-time Ultrasound feedback diaphragm 
Table 2. Comparison of the pulmonary function at pre- and post-intervention in the three groups

$(\mathrm{N}=30)$

\begin{tabular}{|c|c|c|c|c|c|}
\hline & & SDBG & PDBG & UFDBG & $\mathrm{F}(\mathrm{p})$ \\
\hline \multirow{4}{*}{ FVC (L) } & Pre & $3.301 \pm 0.621^{\mathrm{a}}$ & $2.728 \pm 0.312$ & $3.088 \pm 0.641$ & $3.194(.056)$ \\
\hline & Post & $3.298 \pm 0.601$ & $3.039 \pm 0.481$ & $3.222 \pm 0.667$ & \\
\hline & Difference & $-0.011 \pm 0.089$ & $0.310 \pm 0.333$ & $0.141 \pm 0.110$ & $5.487(.010)^{\dagger \ddagger}$ \\
\hline & $\mathrm{t}(\mathrm{p})$ & $0.323(.755)$ & $-3.267(.008)^{*}$ & $-4.038(.003)^{*}$ & \\
\hline \multirow{4}{*}{ FEV1 (L) } & Pre & $2.759 \pm 0.621$ & $2.461 \pm 0.342$ & $2.657 \pm 0.481$ & $1.069(.357)$ \\
\hline & Post & $2.844 \pm 0.581$ & $2.558 \pm 0.291$ & $2.701 \pm 0.354$ & \\
\hline & Difference & $0.079 \pm 0.261$ & $0.111 \pm 0.161$ & $0.042 \pm 0.184$ & $0.338(.716)$ \\
\hline & $\mathrm{t}(\mathrm{p})$ & $-0.941(.374)$ & $-2.456(.032)^{*}$ & $-0.743(.477)$ & \\
\hline \multirow{4}{*}{ MVV (L) } & Pre & $85.811 \pm 26.647$ & $71.533 \pm 18.091$ & $80.727 \pm 17.484$ & $1.296(.289)$ \\
\hline & Post & $87.141 \pm 20.642$ & $83.944 \pm 21.554$ & $87.267 \pm 19.017$ & \\
\hline & Difference & $1.332 \pm 9.108$ & $12.421 \pm 9.527$ & $6.543 \pm 6.391$ & $4.413(.022)^{\dagger \neq}$ \\
\hline & $\mathrm{t}(\mathrm{p})$ & $-0.439(.672)$ & $-4.512(.001)^{*}$ & $-3.235(.010)^{*}$ & \\
\hline \multirow{4}{*}{ RR } & Pre & $47.443 \pm 9.028$ & $45.921 \pm 9.747$ & $46.101 \pm 8.307$ & $0.082(.922)$ \\
\hline & Post & $44.224 \pm 7.762$ & $48.001 \pm 8.642$ & $46.704 \pm 9.821$ & \\
\hline & Difference & $-3.221 \pm 7.452$ & $2.081 \pm 6.599$ & $0.603 \pm 8.361$ & $1.345(.277)$ \\
\hline & $\mathrm{t}(\mathrm{p})$ & $1.298(.230)$ & $-1.094(.297)$ & $-0.227(.825)$ & \\
\hline
\end{tabular}

${ }^{\mathrm{a}} \mathrm{Mean} \pm$ standard deviation

${ }^{*}$ Significant different in the pre-post value $(\mathrm{p}<.05)$

${ }^{\dagger}$ Significant different among the three groups $(\mathrm{p}<.05)$

${ }^{\ddagger}$ Significant different in PDBG compared with SDBG $(\mathrm{p}<.05)$.

SDBG : Self-diaphragm breathing group, PDBG : Passive-diaphragm breathing group, UFDBG : Real-time Ultrasound feedback diaphragm

FVC : Forced vital capacity, FEV1 : Forced expiratory volume at one second, MVV : Maximal voluntary ventilation, RR : Respiration rate

as breathing exercises that help improve the ventilation capabilities as well as the pulmonary function and cough abilities by applying respiratory support to spinal cord injury patients or people with neuropathy disorders who use excessive respiratory support[5]. Lee[16] reported improvement in the pulmonary functions and strength of adults with a head forward posture (Especially VC) by learning the correct contraction of the diaphragm. Despite the results of these studies, none of the measures of the lung capacity showed a significant improvement in the self-impaired group.
Correct contraction of the diaphragm is important during diaphragm breathing. On the other hand, many older patients or patients with respiratory diseases are more accustomed to the use of costal respiration and accessory breathing muscles than to the use of the diaphragm. Therefore, it is important for the subjects to recognize the precise contraction of the diaphragm so that they can perform the exercise. To detect the contraction of the dia phragm accurately, this study used real-time ultrasound imaging equipment to identify the contraction of the diaphragm directly[17]. Sartori et al.[18] reported an 
Table 3. Comparison of the diaphragm thickness at pre- and post-intervention in the three groups

\begin{tabular}{|c|c|c|c|c|c|}
\hline & & SDBG & PDBG & UFDBG & $\mathrm{F}(\mathrm{p})$ \\
\hline \multirow{4}{*}{$\begin{array}{l}\text { Inspiration } \\
\text { (mm) }\end{array}$} & Pre & $1.699 \pm 0.181^{\mathrm{a}}$ & $1.631 \pm 0.204$ & $1.581 \pm 0.182$ & $0.955(.397)$ \\
\hline & Post & $1.702 \pm 0.119$ & $1.674 \pm 0.147$ & $1.581 \pm 0.188$ & \multirow{3}{*}{$0.046(.955)$} \\
\hline & Difference & $-0.011 \pm 0.212$ & $0.041 \pm 0.267$ & $0.012 \pm 0.271$ & \\
\hline & $\mathrm{t}(\mathrm{p})$ & $-0.097(.925)$ & $-0.466(.651)$ & $-0.058(.955)$ & \\
\hline \multirow{4}{*}{$\begin{array}{l}\text { Expiration } \\
(\mathrm{mm})\end{array}$} & Pre & $1.339 \pm 0.141$ & $1.311 \pm 0.188$ & $1.327 \pm 0.191$ & $0.047(.955)$ \\
\hline & Post & $1.391 \pm 0.151$ & $1.347 \pm 0.161$ & $1.254 \pm 0.137$ & \multirow{3}{*}{$0.436(.651)$} \\
\hline & Difference & $0.051 \pm 0.222$ & $0.042 \pm 0.231$ & $-0.069 \pm 0.221$ & \\
\hline & $\mathrm{t}(\mathrm{p})$ & $-0.713(.496)$ & $-0.537(.602)$ & $1.052(.320)$ & \\
\hline \multirow{4}{*}{$\begin{array}{c}\text { Thickness } \\
\text { ratio } \\
(\%)\end{array}$} & Pre & $27.486 \pm 12.841$ & $25.292 \pm 13.437$ & $20.100 \pm 14.661$ & $0.747(.483)$ \\
\hline & Post & $23.511 \pm 13.936$ & $24.761 \pm 12.792$ & $27.367 \pm 16.204$ & \multirow{3}{*}{$1.189(.320)$} \\
\hline & Difference & $-3.982 \pm 15.891$ & $-0.531 \pm 19.974$ & $7.267 \pm 11.431$ & \\
\hline & $\mathrm{t}(\mathrm{p})$ & $0.751(.474)$ & $0.092(.928)$ & $-2.012(.075)$ & \\
\hline
\end{tabular}

${ }^{\mathrm{a}}$ Mean \pm standard deviation

${ }^{*}$ Significant different in the pre-post value $(\mathrm{p}<.05)$

${ }^{\dagger}$ Significant different among the three groups $(\mathrm{p}<.05)$

SDBG : Self-diaphragm breathing group, PDBG : Passive-diaphragm breathing group, UFDBG : Real-time ultrasound feedback diaphragm

improved pulmonary function in patients with fibrotic cysts after being provided feedback on the diaphragm contractions through real-time ultrasound imaging. Lee[19] reported that ultrasound-based feedback breathing training helped improve the pulmonary function of stroke patients.

This study hypothesized that when visual feedback is applied to correct a contraction of the diaphragm, there is a significant difference in pulmonary function improvement and diaphragm contraction rate compared to general diaphragm breathing or resistance diaphragm breathing. Diaphragm breathing training methods using real-time ultrasound imaging feedback are effective in improving the maximum ventilation capacity as well as the pulmonary function. On the other hand, it did not show any significant differences compared to the general diaphragm breathing training method. On the other hand, resistive-diaphragm breathing training is a common diaphragm-breathing method that involves the simultaneously application of resistance during breathing. Not only was. there significant improvement in pulmonary function and an increase in the maximum ventilation post- and preintervention, there was also a higher variation compared to the general breathing training groups. Although this was a short term intervention, resistive-diaphragm breathing training could lead to an expansion of the diaphragm and activation of the expiration muscles. These results, unlike the other groups, can be seen only in the result of the increase in FEV1 (Forced expiratory volume at 1 second) in pre- and post- intervention. Considering that all participants in the study were young adults and that the subjects were already familiar with diaphragm-breathing, it is believed that the impact of resistive-diaphragm breathing on breathing resistance was greater than that achieved by just providing visual feedback. The effect of 
resistive-diaphragm breathing training can be understood through the results of previous studies. Enright et al.[20] reported that a group of breathing exercises that provided resistance to inspiration showed an improvement in breathing. Liaw et al.[21] performed resistive-diaphragm breathing exercise on patients with spinal cord injury, and reported an increase in pulmonary function. Kim and Shin [22] suggested that high intensity intermittent training can result in significant improvement in the cardiopulmonary capacity of canoe and kayak paddlers. To support these studies, resistive-diaphragm breathing training can be proposed as an effective respiration training method to improve the pulmonary function and maximum vital capacity.

This study had some limitations. First, the duration of intervention in the breathing training method was short, i.e., did not maximize the effect of the intervention. Second, the total number of participants and the 10 in each group were insufficient to generalize the results. Third, although healthy and young adults in their 20's can recognize their diaphragm breathing relatively easily, people with respiratory diseases or older adults may have other issues. Different results may be obtained if these exercises are performed on elderly people or patients with respiratory disease. Therefore, further studies will be needed on patients of various ages and disease states by ensuring a sufficient intervention period and number of subjects.

\section{Conclusion}

This study compared the effects of various methods of diaphragm breathing training on the pulmonary function and the contraction of the diaphragm. The results revealed significant improvement in the forced vital capacity and maximal voluntary ventilation over other diaphragm breathing training groups. This means that resistivediaphragm breathing training can help increase the pulmonary function in a short period of time. Therefore, resistive-diaphragm breathing training can lead to significant improvements in the pulmonary function of patients that do not have reduced or limited respiratory functions in clinical practice. Therefore, clinical trials should consider the impact of motor loads and resistance on the planning of an intervention program.

\section{Reference}

[1] Fugl-Meyer AR, Linderholm $\mathrm{H}$, Wilson $\mathrm{AF}$, et al. Restrictive ventilatory dysfunction in stroke: its relation to locomotor function. Scand J Rehabil Med Suppl. 1983;9:118-24.

[2] Urfy MZ, Suarez J. Breathing and the nervous system. Handb Clin Neurol. 2014;119:241-50.

[3] Frownfelter D, Stevens K, Massery M, et al. Do abdominal cutouts in thoracolumbosacral orthoses increase pulmonary function? Clin Orthop Relat Res. 2014a;472(2):720-6.

[4] Kisner C, Colby LA, Borstad J, et al. Therapeutic Exercise: Foundations and Techniques(7th ed). USA. F.A. Davis. 2017.

[5] Frownfelter D, Dean E. Cardiovascular and Pulmonary Physical Therapy - E-Book: Evidence to Practice(5 th ed). USA. Elsevier Health Sciences. 2014b.

[6] Cha HG, Choe YW, Kim MK, et al. The Effects of Air Stacking Exercise on Pulmonary Function in Elderly Adults. J Korean Soc Phys Med. 2016;11(4):55-64.

[7] Carr M, Jones J. Physiological effects of exercise on stroke survivors. Top Stroke Rehabil. 2003;9(4):57-64.

[8] Back EM. A study on the effects of using musical rhythm pattern to improve speech of patients suffering from brain damage. Master's Degree. Sookmyung Women's University. 2003

[9] Fried R. The Psychology and Physiology of Breathing: In Behavioral Medicine, Clinical Psychology, and Psychiatry. USA. Springer US. 1993.

[10] Moodie L, Reeve J, Elkins M, et al. Inspiratory muscle 
training increases inspiratory muscle strength in patients weaning from mechanical ventilation: a systematic review. J Physiother. 2011;57(4):213-21.

[11] Weiner P, Magadle R, Beckerman M, et al. Comparison of specific expiratory, inspiratory, and combined muscle training programs in COPD. Chest. 2003;124(4):1357-64.

[12] Dursun N, Dursun E, Kilic Z, et al. Electromyographic biofeedback-controlled exercise versus conservative care for patellofemoral pain syndrome. Arch Phys Med Rehabil. 2001;82(12):1692-5.

[13] Kwon NH, Lee HO, Park DJ, et al. The Use of Real-Time Ultrasound Imaging for Feedback during Abdominal Hollowing. J Korean Soc Phys Med. 2011;6(3):303-10.

[14] Nam SJ. Effects of Diaphragmatic Breathing Training Using Real-time Ultrasonography on Chest Function in Young Females with Limited Chest Mobility. Master's Degree. Baekseok University. 2017.

[15] Sarwal A, Walker FO, Cartwright MS, et al. Neuromuscular ultrasound for evaluation of the diaphragm. Muscle Nerve. 2013;47(3):319-29.

[16] Lee JC. The Effects of Abdominal Respiratory Exercise by Forward Head Posture Adult's Respiratory Function and Respiratory Muscle Strength. Master's Degree.
Daegu University. 2015.

[17] Hodges PW. Ultrasound imaging in rehabilitation: just a fad? J Orthop Sports Phys Ther. 2005;35(6):333-7.

[18] Sartori R, Barbi E, Poli F, et al. Respiratory training with a specific device in cystic fibrosis: a prospective study. J Cyst Fibros. 2008;7(4):313-9.

[19] Lee JH. The Effect of Pulmonary Function in the Stroke Patients after feedback breathing exercise. Gyeongsan. Master's Degree. Daegu University. 2008.

[20] Enright SJ, Unnithan VB, Heward C, et al. Effect of high-intensity inspiratory muscle training on lung volumes, diaphragm thickness, and exercise capacity in subjects who are healthy. Phys Ther. 2006;86(3): 345-54.

[21] Liaw MY, Lin MC, Cheng PT, et al. Resistive inspiratory muscle training: its effectiveness in patients with acute complete cervical cord injury. Arch Phys Med Rehabil. 2000;81(6):752-6.

[22] Kim AR, Shin WS. Research Article : Open Access ; Effects of High-intensity Intermittent Training and Moderate-intensity Training on Cardiopulmonary Capacity in Canoe and Kayak Paddlers during 8 weeks. J Korean Soc Phys Med. 2014;9(3):307-14. 
